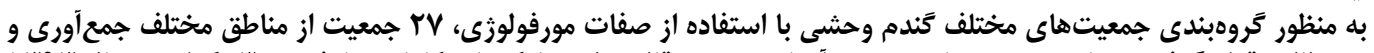

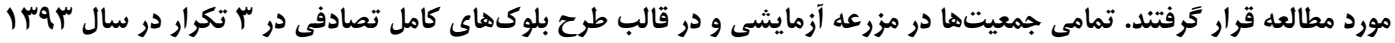

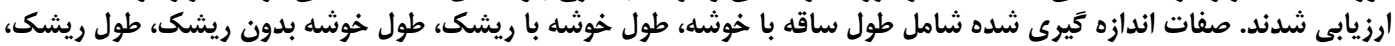

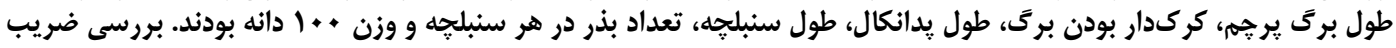

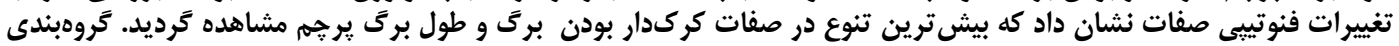

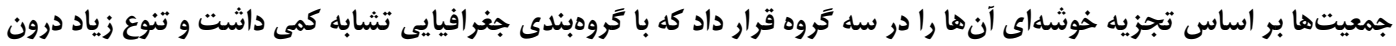

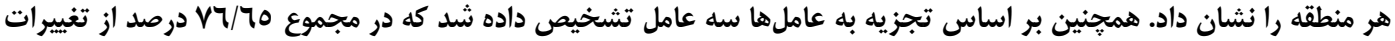

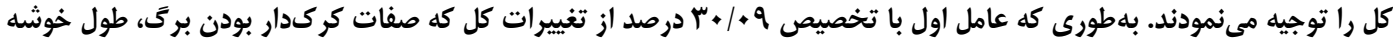

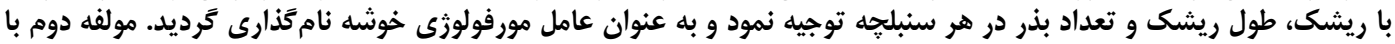

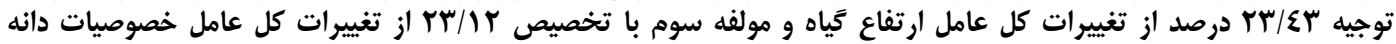

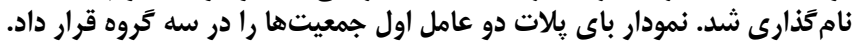

وازههاى كليد: كَندم وحشى، تجزيه خوشهاى، تجزيه به عاملها

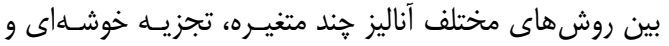

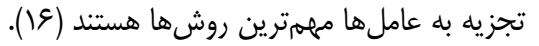

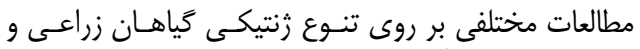

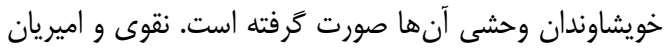

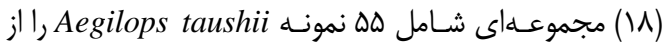

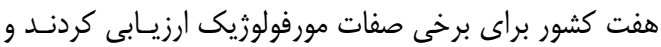

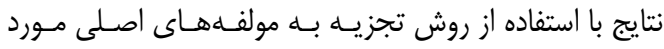

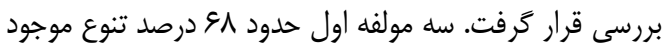

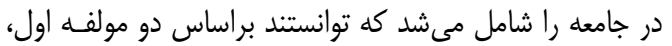
دو زير گونسه

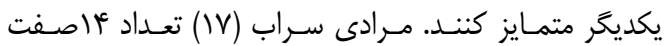

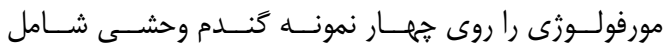
T. arararticum, T. urartu ، T. thaudar ، T. boeticum بررسى و دادهها را از طريق روشهاى آمارى تجزيه و تحليـل

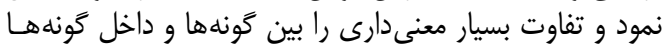

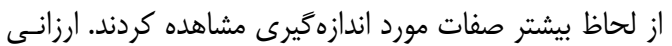

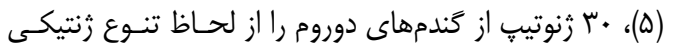

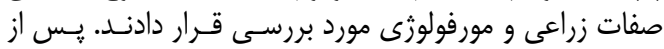

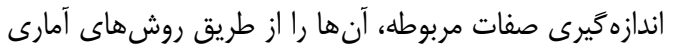

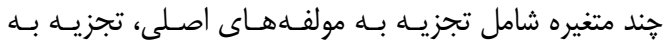

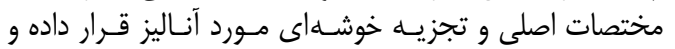

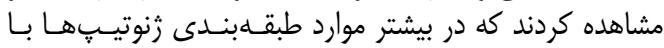

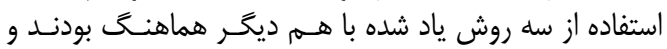

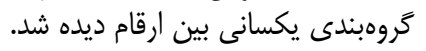

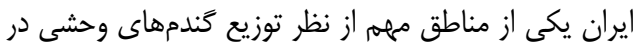

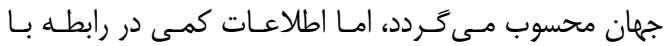

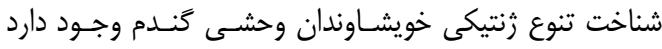

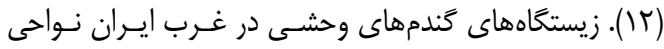

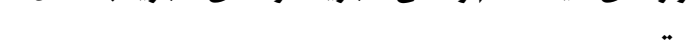
كَندم يكى از مهمهترين گياهان زراعى در جهـان اسـت كـه

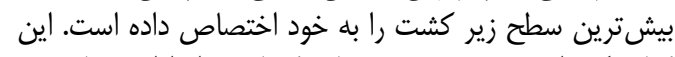

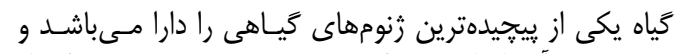

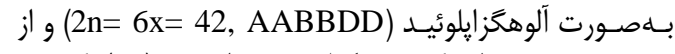

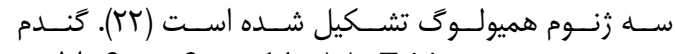
كون 2x = 14, AA) Triticum monococcum

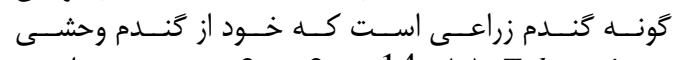
(2n = 2x = 14, AA) T. boeoticum

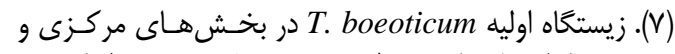

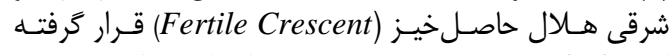

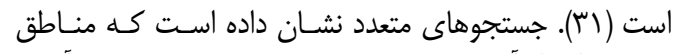

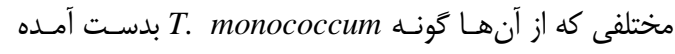

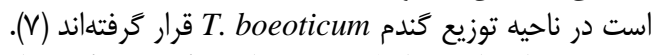

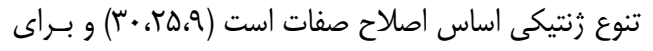

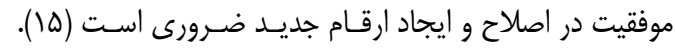

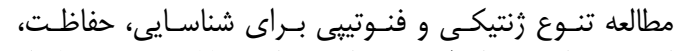

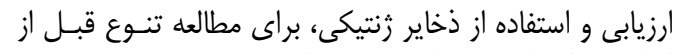

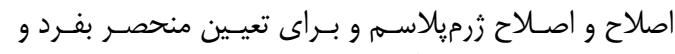

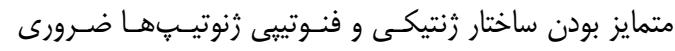

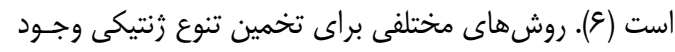

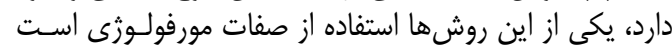

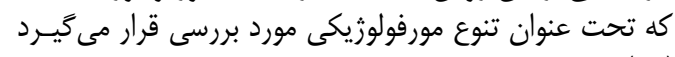

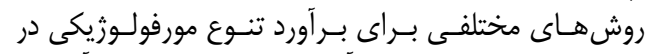

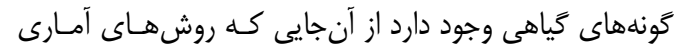

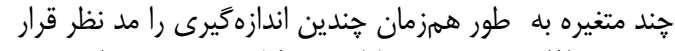

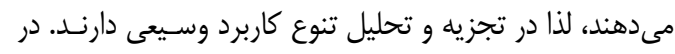


مختلف و معيار هاى فاصله متفاوت انجام شد و بلمنظور تعيين

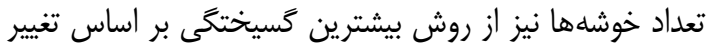

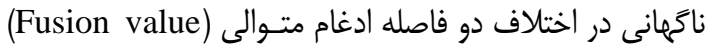

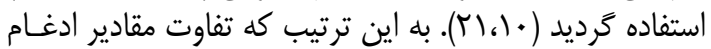

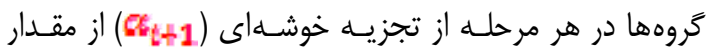

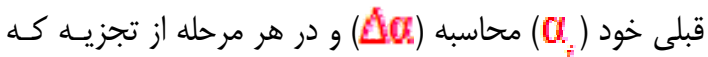

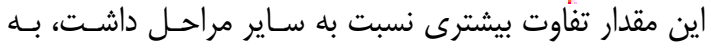
عنوان نقطه برش دندروكرام انتخاب و تعداد خوشهها براب بر مبناى

$\Delta \alpha=\alpha_{i+1}-\alpha_{i}$

آن مشخص شدند (رابطه ().).

رابطه (1)

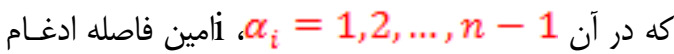

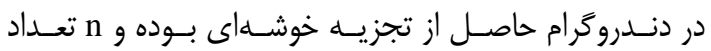

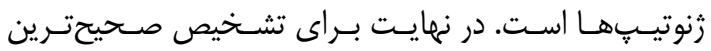

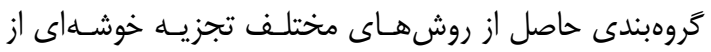

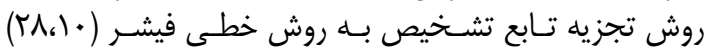

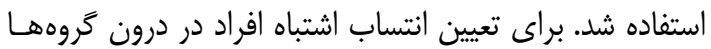

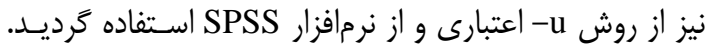

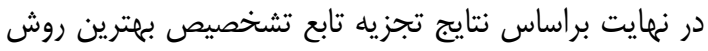

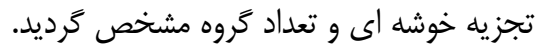

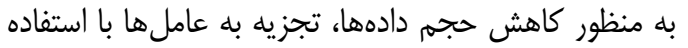

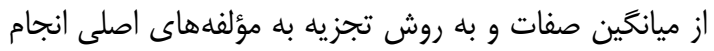

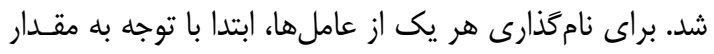

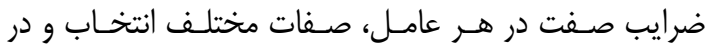

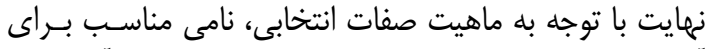

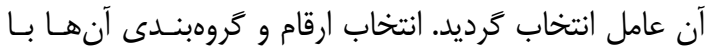

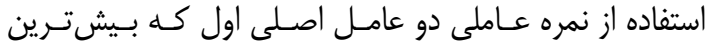

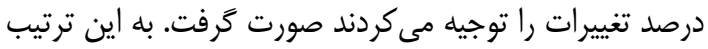

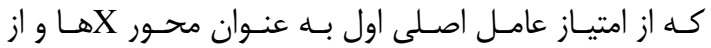

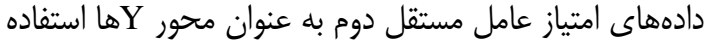

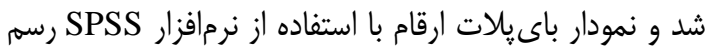

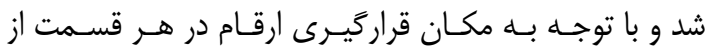

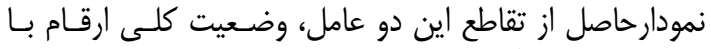

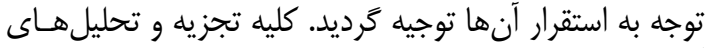

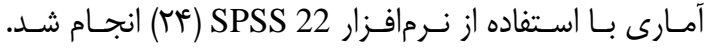

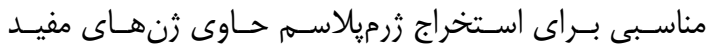

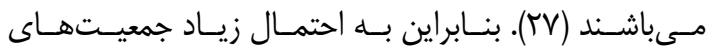
T. T. boeoticum

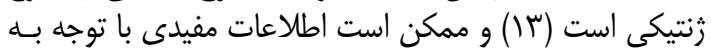

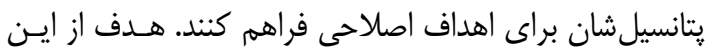

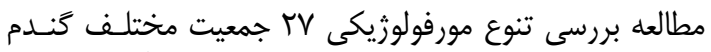

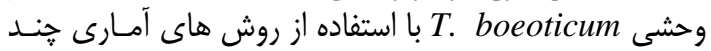
متغيره است.

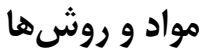

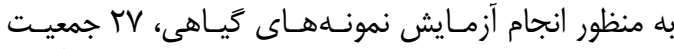

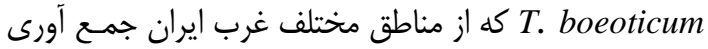

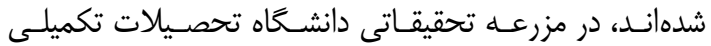

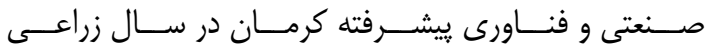

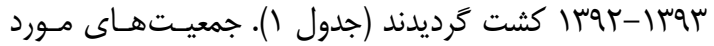

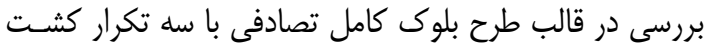

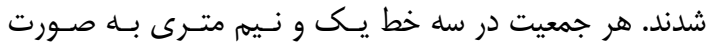

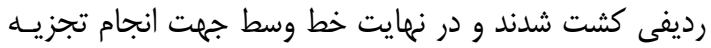

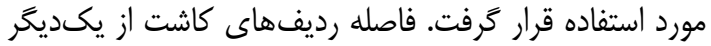

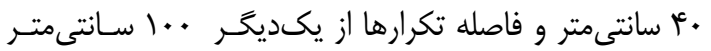

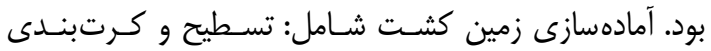

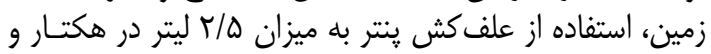

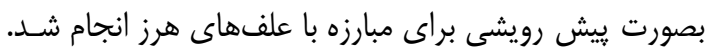

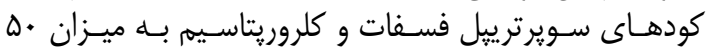

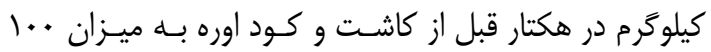

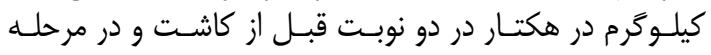

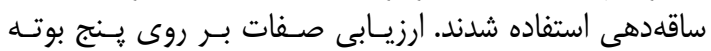

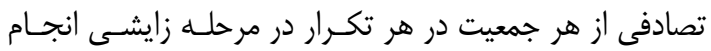

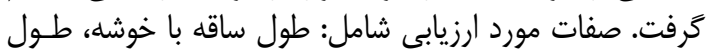

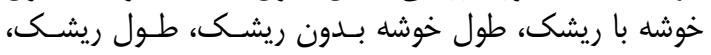

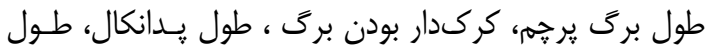

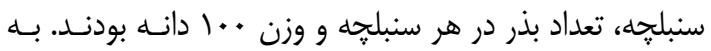

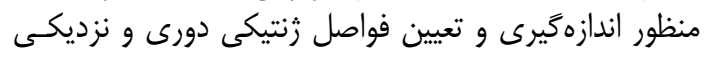

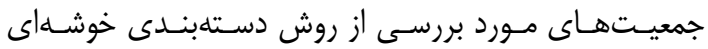
استفاده شد. به اين صورت كه تجزيه خوشهاى بـ بـا روشهــاى درى 
Table1. Geographical distribution of studied populations

جدول ا - يراكنش جغرافيايى جمعيتهاى مورد مطالعه

\begin{tabular}{|c|c|c|c|c|c|c|c|}
\hline محل جمع آورى & استان & 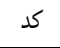 & رديف. & محل جمع آورى & استان & 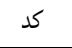 & رديف \\
\hline كوزران & كرمانشاه & $\mathrm{C} 2$ & 10 & زاغه & ل لرستان & $\mathrm{A} 1$ & 1 \\
\hline 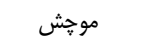 & كردستان & $\mathrm{C} 3$ & 19 & دولتشاه & 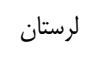 & A3 & r \\
\hline كنجى & كردستان & $\mathrm{C} 4$ & iv & روستاى هزار خانى & ل ل لرستان & A4 & r \\
\hline كامياران & كردستان & $\mathrm{C} 5$ & $M$ & نورآباد & ل ل لرستان & A6 & r \\
\hline بلبان آباد & كردستان & C6 & 19 & ر ازان & ل ل لرستان & A7 & $\Delta$ \\
\hline إلنغان & كردستان & $\mathrm{C} 7$ & r. & جاده فيروزآباد خرم آباد & 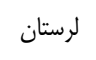 & A10 & 9 \\
\hline كامياران & كردستان & $\mathrm{C} 9$ & r & سفيد دشت & 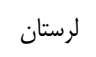 & A11 & $v$ \\
\hline قشلاق مريوان & كردستان & $\mathrm{C} 10$ & r & ن ن امشخص & 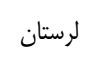 & $\begin{array}{r}\mathrm{A} 7 / 1 \\
1\end{array}$ & $\wedge$ \\
\hline سقز & كردستان & $\mathrm{C} 11$ & r & ده سفيد & كرمانشاه & B1 & 9 \\
\hline سرو آباد & كردستان & $\mathrm{C} 12$ & re & هرسين & كرمانشاه & B2 & 1. \\
\hline روستاى اشداله & آذربايجان شرقى & D1 & To & كرمانشاه & كرمانشاه & B3 & 11 \\
\hline اهر & آذربايجان شرقى & D2 & rq & ياوه & كرمانشاه & B4 & ir \\
\hline \multirow[t]{2}{*}{ 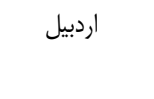 } & اردبيل & E1 & tr & روستاى خسرو آباد & كرمانشاه & B5 & r \\
\hline & & & & دوراهى موجش هـنـدج & كردستان & $\mathrm{C} 1$ & 15 \\
\hline
\end{tabular}

سانتى متر ارتفاع داشت. اين تنوع بالا در ارتفاع بوته مسىتوانـــ

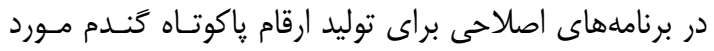

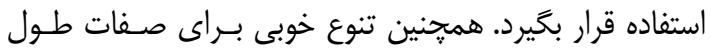

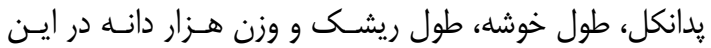

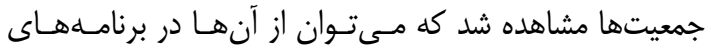

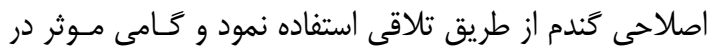
اصلاح گَندم برداشت.

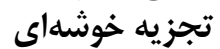

گروهبندى ارقام با روشهاى

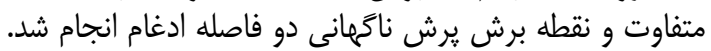

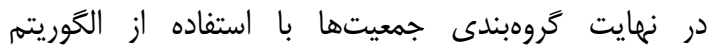
UPGMA

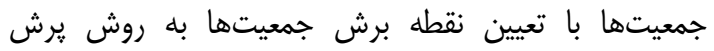

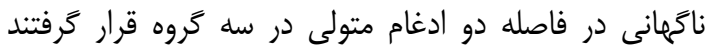

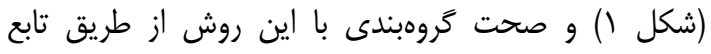

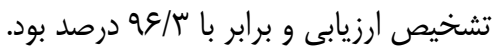

\section{نتايج و بحث \\ آمارههاى توصيفى كل جمعيت}

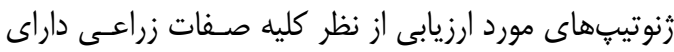

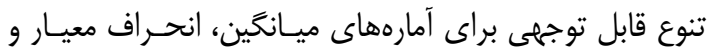

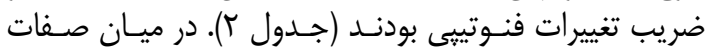

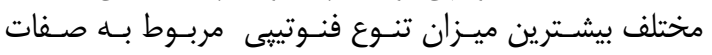

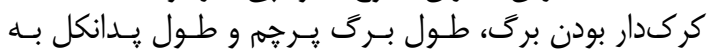

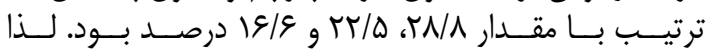

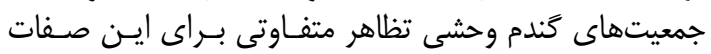

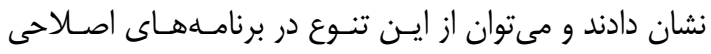

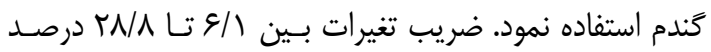

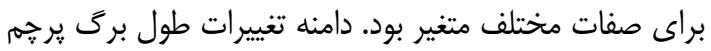

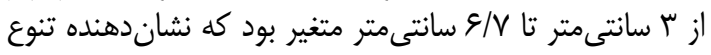

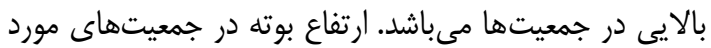

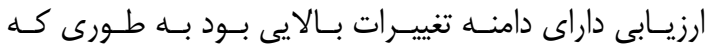

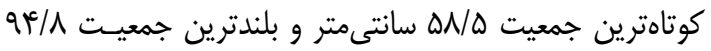

جدول r-boeoticum يارامترهاى آمار توصيفى مربوط به صفات مورد بررسى در جمعيتهاى كَندم وحشى Table 2. Descriptive statistical parameters of studied traits in populations of wild wheat T. boeoticum

\begin{tabular}{|c|c|c|c|c|c|c|c|c|c|c|}
\hline & $\begin{array}{c}\text { ارتفاع } \\
\text { بوته } \\
(\mathrm{cm})\end{array}$ & 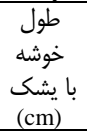 & $\begin{array}{c}\text { طول خوشن } \\
\text { بدون } \\
\text { (cm) } \\
\end{array}$ & $\begin{array}{l}\text { ريشكل } \\
(\mathrm{cm})\end{array}$ & طول برگ & كر كدار & $\begin{array}{c}\text { طدانكال } \\
(\mathrm{cm})\end{array}$ & $\begin{array}{l}\text { سنبل جه } \\
\text { (mm) } \\
\text { (mm) }\end{array}$ & سنداد بذر & $\begin{array}{c}\text { وزن } \\
1 .+. \\
\text { (gr) } \\
\end{array}$ \\
\hline ميانخين & VE/FT & $I V / r V$ & $\Lambda / \& \Lambda$ & N/AT & $f / r r$ &.$/ 94$ & $M F / D$ & $1 / 9 V$ & $1 / 9 \pi$ & سז/T \\
\hline انحراف معيار & $q / \%$. & $1 / 99$ & $1 / 10$ & $1 / 1 F$ &.$/ 91$ & . & $\Delta / V^{c}$ &.$/ 1$ & $\cdot / r$ & $\cdot / H^{F}$ \\
\hline واريانس & $N / \Delta$. & r/N & س & Tr/T & .199 & $\cdot / \cdot V$ & F & $\cdot 1 \cdot 1$ & $\cdot 1 \cdot t^{4}$ & $\cdot 1 \cdot \Delta$ \\
\hline مينيمم & $\Delta N / \Delta$. & $\mid r / r$. & $\mathrm{V} / \ldots$ & $\Delta / V$. & 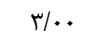 &.$/$. & $r / q$. & $1 / 48$ & $1 / r \Delta$ & $1 / \wedge 1$ \\
\hline ماكزيمم & $9 \% / \Lambda$. & $r t /$. & $11 / .$. & $1 \cdot / v$ & $s / v$. & $1 / \cdot$ & $\mathrm{Fr} / \mathrm{.}$ & $1 / 19$ & $r / .$. & $r / \& \Lambda$ \\
\hline ضريب تغييرات & I/IT & $m / l$ & r/l & س/ & D/TT & $\Lambda / T \Lambda$ & $q / 19$ & $1 / 9$. & $r / I T$ & $N / 1$. \\
\hline
\end{tabular}


نشاندهنده تفاوت آنها با ساير جمعيتهاى كروههاى ديخر و و

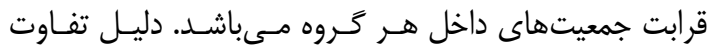

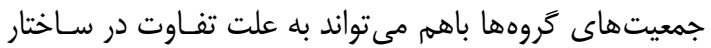

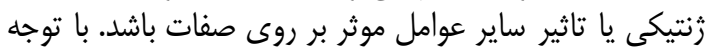

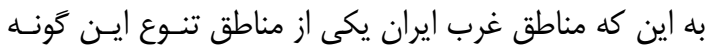

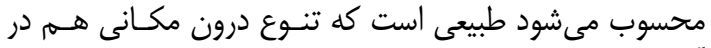

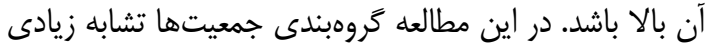

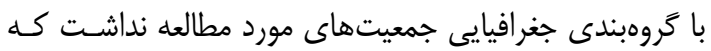

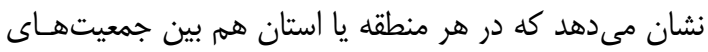

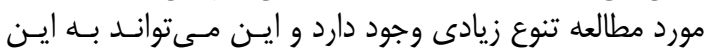

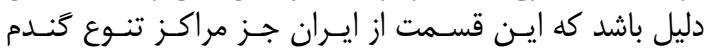
مىباشد.

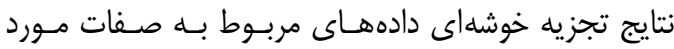

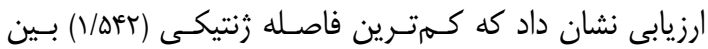

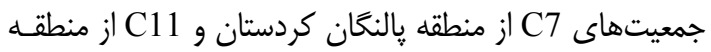

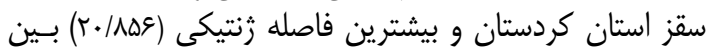

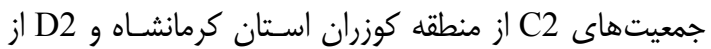

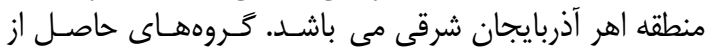

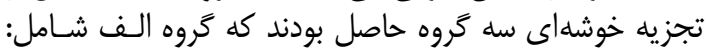

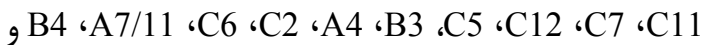

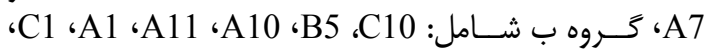

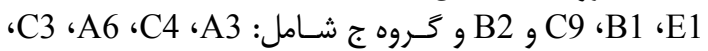

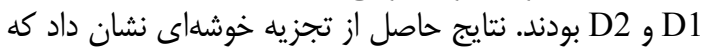

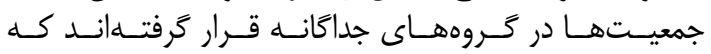

جدول بـ ميانگين و درصد انحراف از ميانگَين كل گروه ها براى صفات مختلف كَندمهاى وحشى Table 3.The average and percent deviation from the total average of groups for different traits of wild wheat

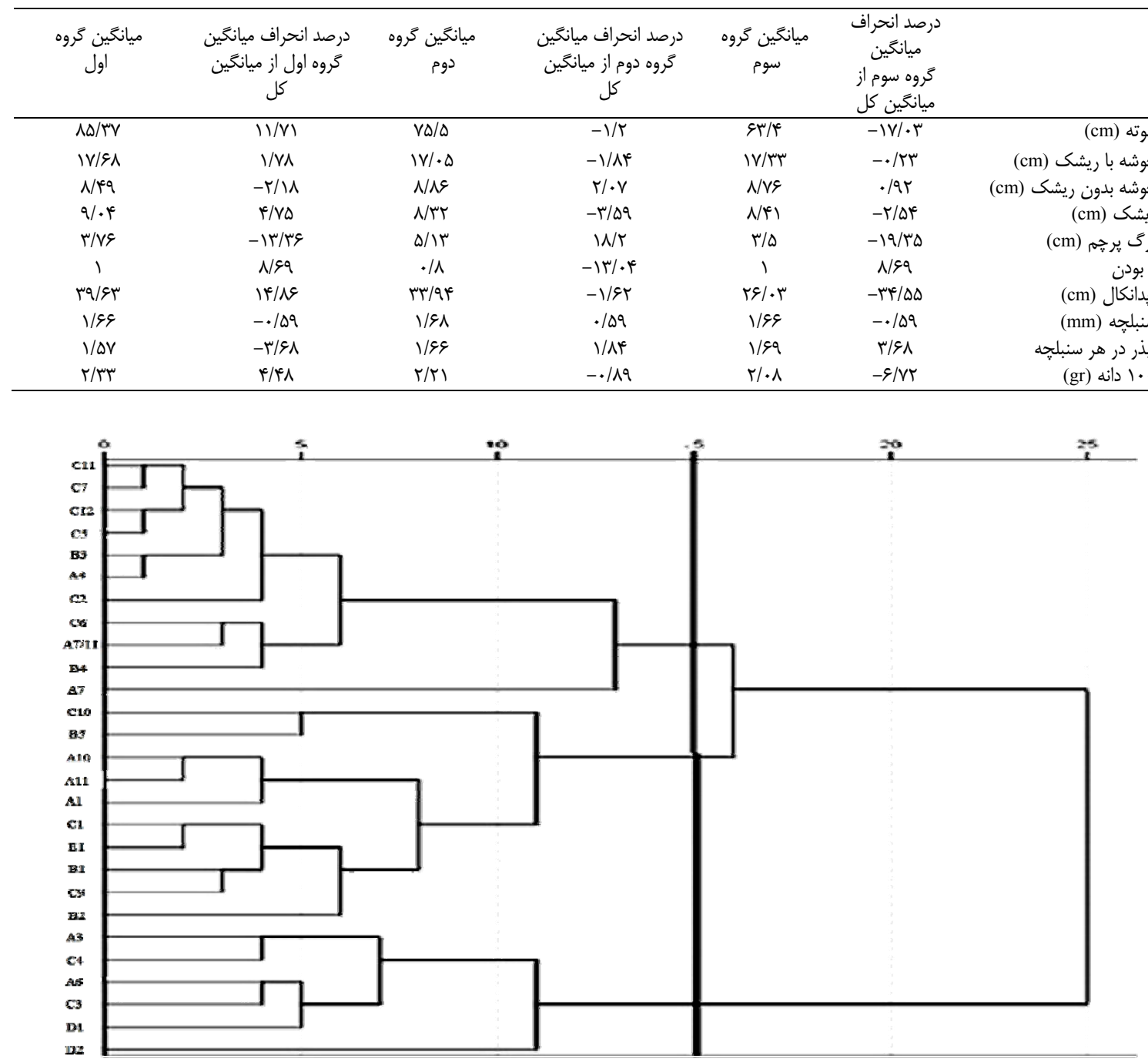

شكل 1- دندروگرام حاصل از تجزيه خوشهاى

Figure1. Dendrogram of cluster analysis 


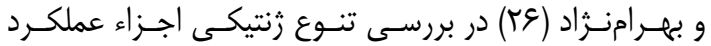

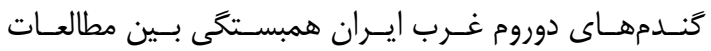

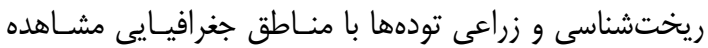
نكردند.

تجزيه به عاملها

در تجزيه به عاملها مئهوان بهان بهطور موفقيتآميزى شمار

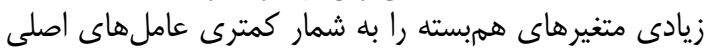

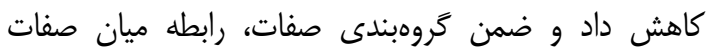

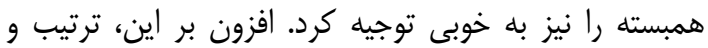

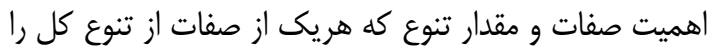

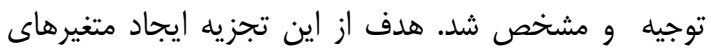

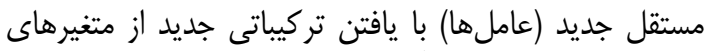

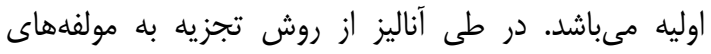

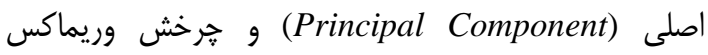

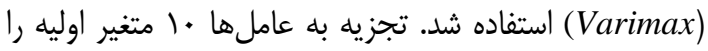

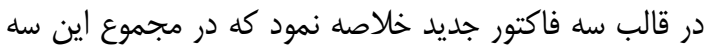

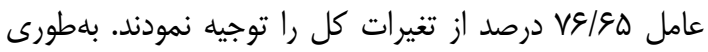

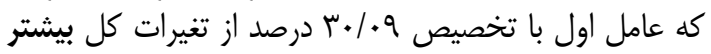

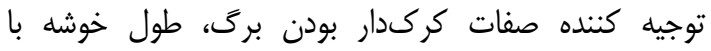

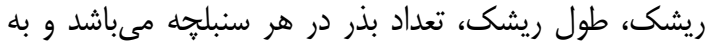

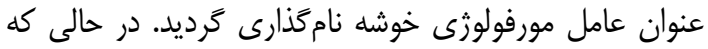

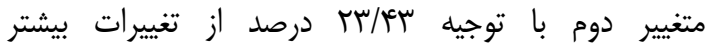

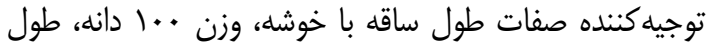

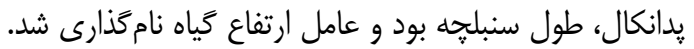

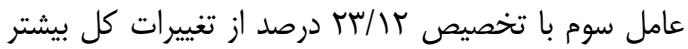

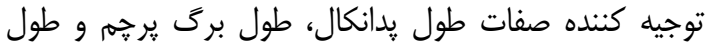

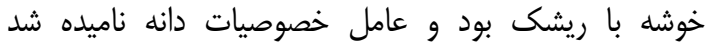

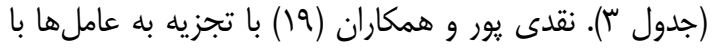

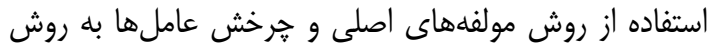

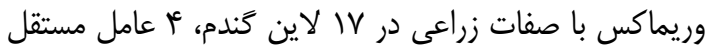

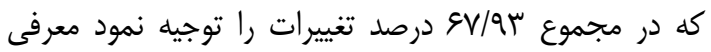

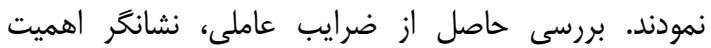

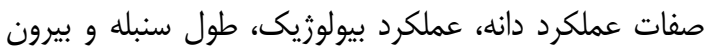

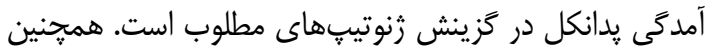

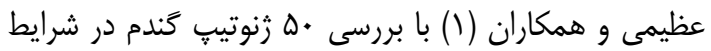

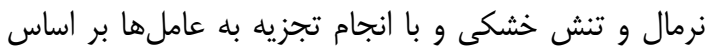

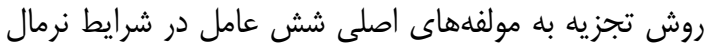

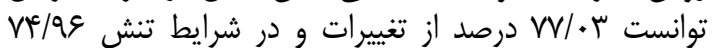
درصد از تغييرات كل را توجيه نمود.

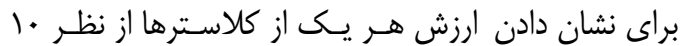

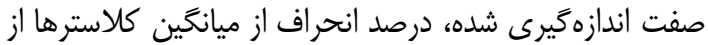

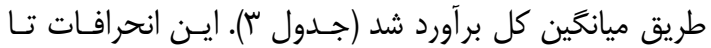

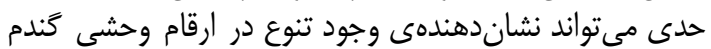

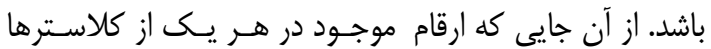

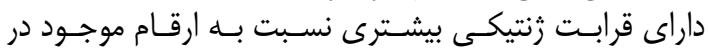

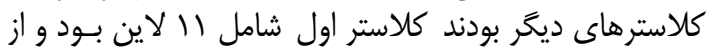

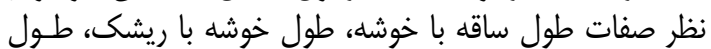

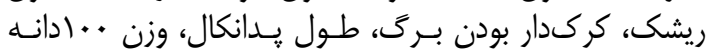

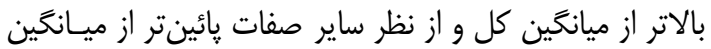

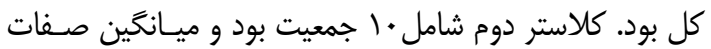

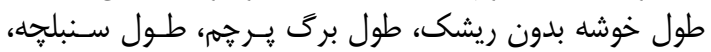

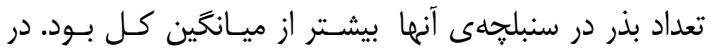

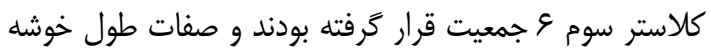

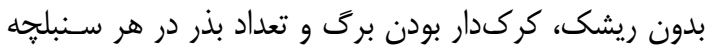

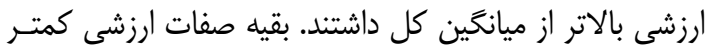

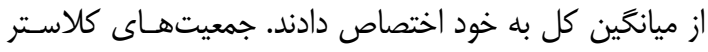

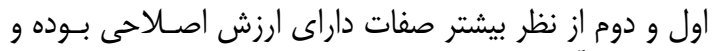

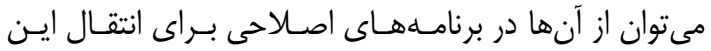

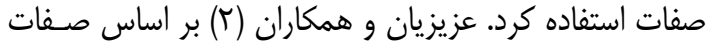

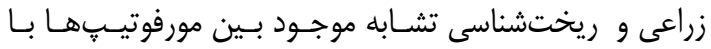

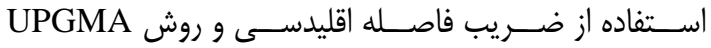

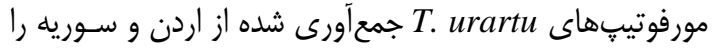

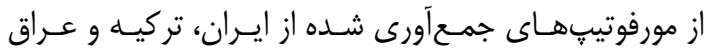

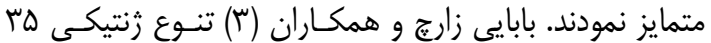

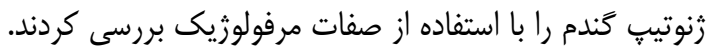

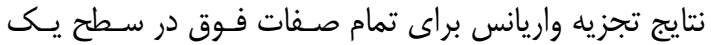

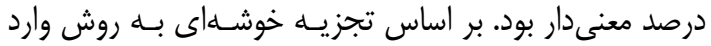

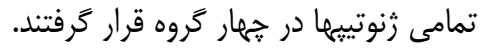

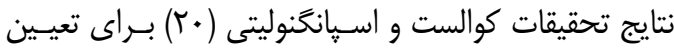

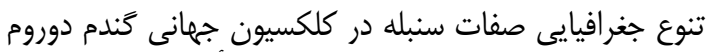

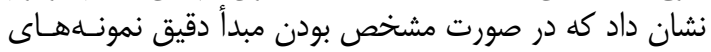

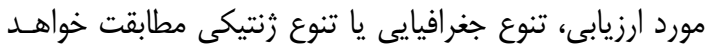

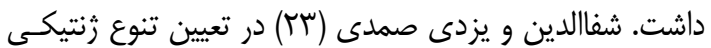

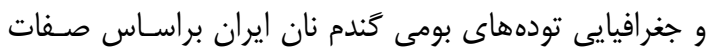

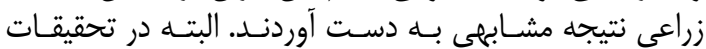

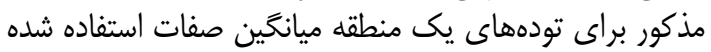

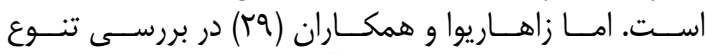

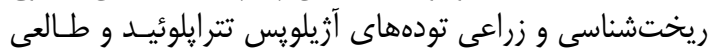


Table 4. Factor analysis for studied traits

جدول ع- ت تجزيه به عاملها براى صفات مورد مطالعه

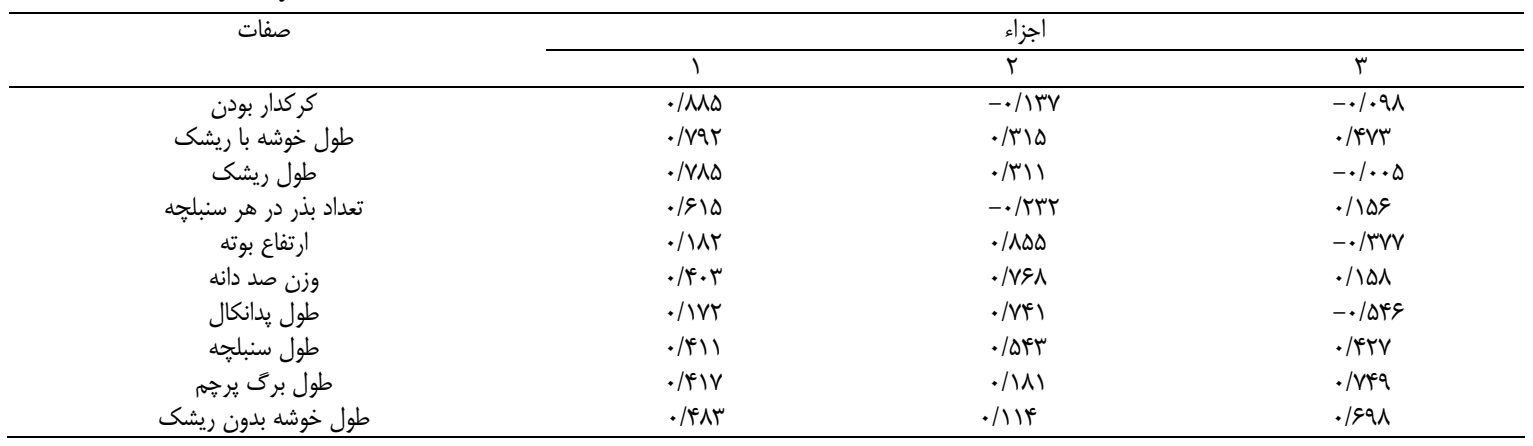

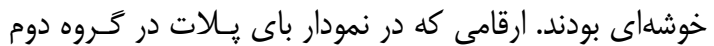

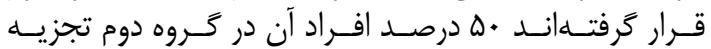

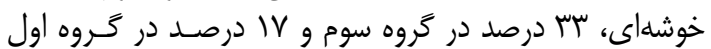

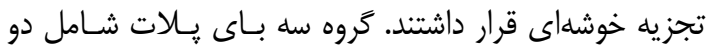

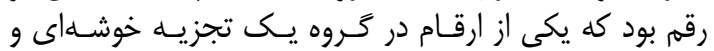

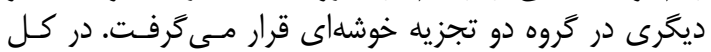

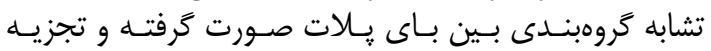

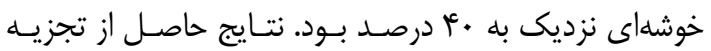

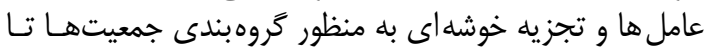

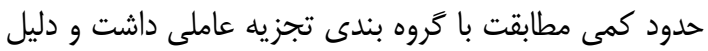

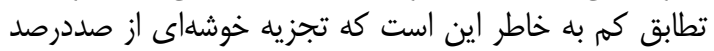

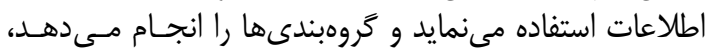

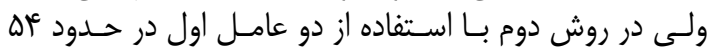

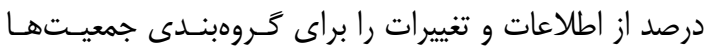

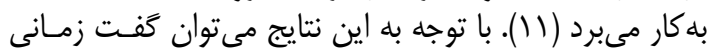

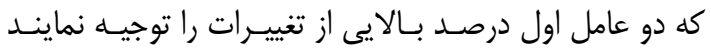

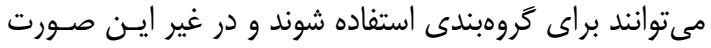

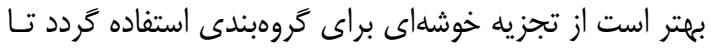

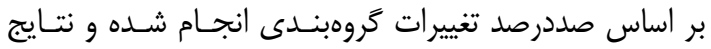
بهترى به دست آيد.
با توجه بهاين كه در تجزيه به عاملهــا، عوامـل مسـتقل و

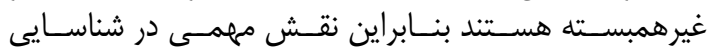

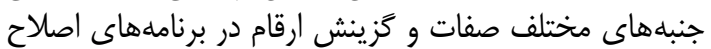

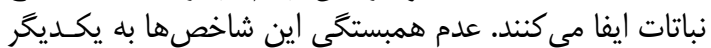

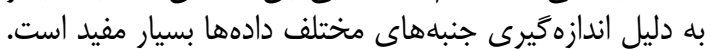

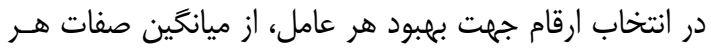

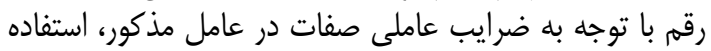

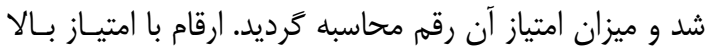

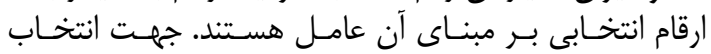

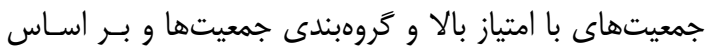

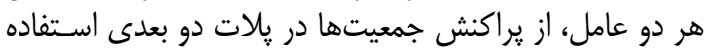

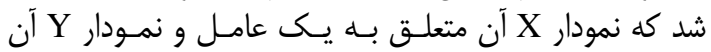

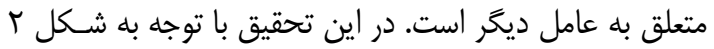

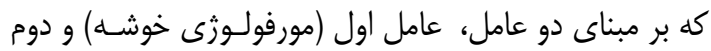

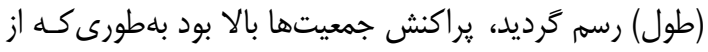

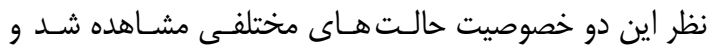

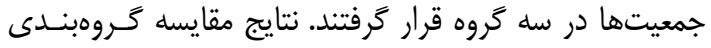

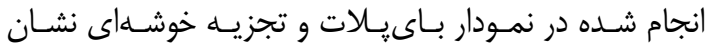

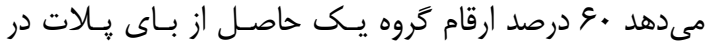

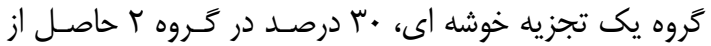

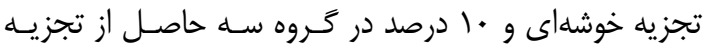




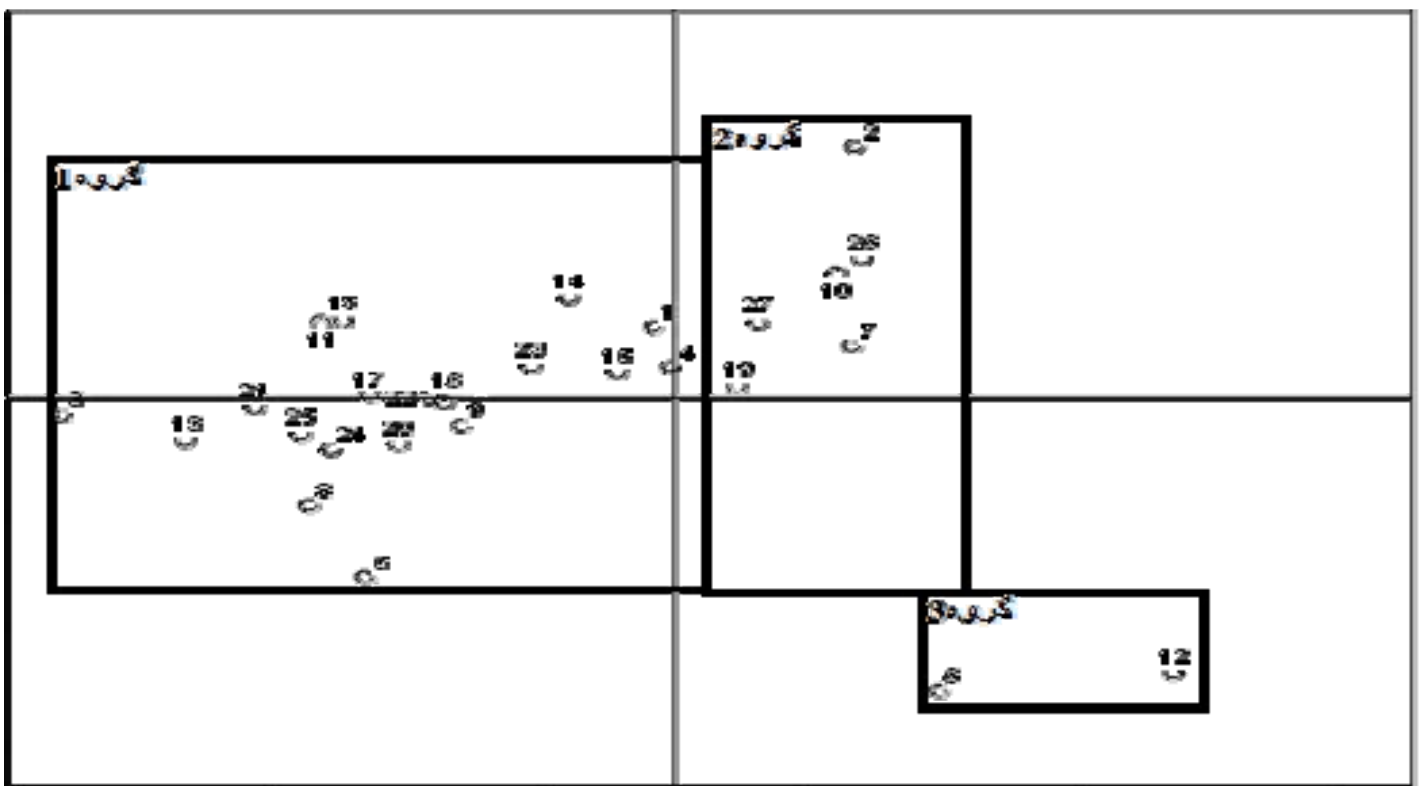

شكل r- نمودار يراكنش جمعيتهاى مورد برررسى بر اساس باى يلات دو عامل اول

Figure 2. Scatter plot of studied populations based on biplot of two first factors

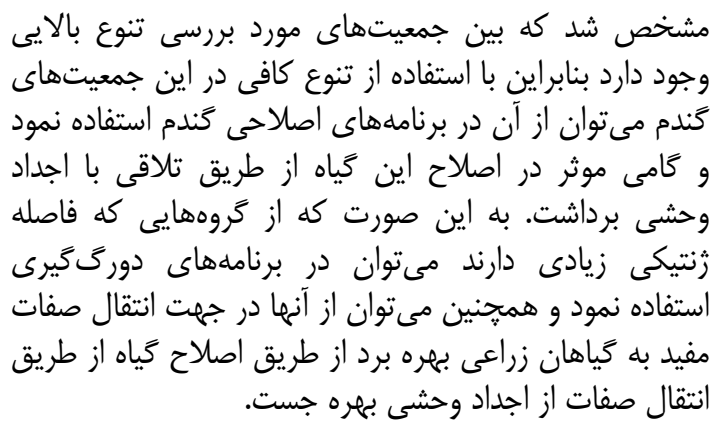

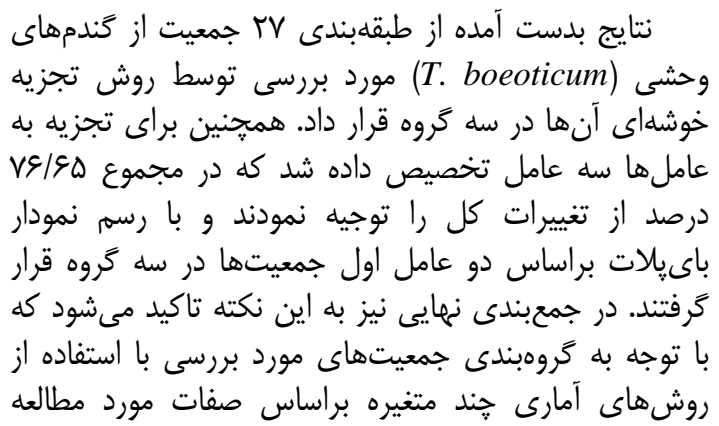

1. Azimi, M., M. Khodarahmi and M.R. JalalKamali. 2012. Evaluation of some important agronomic characteristics in spring bread wheat genotypes under terminal drought stress and non-stress conditions. Journal of Agronomy and Plant Breeding, 8: 175-193 (In Persian).

2. Azizian, A., B. Yazdi Samadi, J. Mozafari, A.A. Shahnejat Boshehri and M.R. Naghavi. 2014. Genetic diversity of diploid wheat (Triticum urartu) using morphological traits and RAPD markers. Journal of Plant Products Research, 21: 149-166 (In Persian).

3. Babaei, Z.M.J., M.H. Fotokian and S. Mahmoodi. 2013. Evaluation of genetic diversity of wheat (Triticum aestivum L.) genotypes for morphological traits using multivarite analysis methods. Journal of crop breeding, 5: 85-98 (In Persian).

4. El-Hendawy, S.E., Y. Hu, G.M. Yakout, A.M. Awad, S.E. Hafiz and U. Schmidhalter. 2005. Evaluating salt tolerance of wheat genotypes using multiple parameters. European journal of agronomy, 22: 243-253.

5. Faraahani, E. and A. Arzani. 2009. Assessment of genetic diversity in durum wheat genotypes with multivariate statistical analysis. Electronic Journal of Crop Production, 1: 51-64 (In Persian).

6. Franco, J., J. Crossa, J. M. Ribaut, J. Bertran, M.L. Warburton and M. Khairallah. 2001. A method for combining molecular markers and phenotypic attributes for classifying plant genotypes. Theoretical and Applied Genetics, 103: 944-952.

7. Harlan, J.R. and D. Zohary. 1996. Distribution of wild wheat and barley. Science, 153: 1074-1080.

8. Houshmand, S., A. Arzani., S.A.M. Maibody and M. Feizi. 2005. Evaluation of salt-tolerant genotypes of durum wheat derived from in vitro and field experiments. Field Crops Research, 91: 345-354.

9. Huang, X.Q., A. Boerner, M.S. Roeder and M.W. Ganal. 2002. Assessing genetic diversity of wheat (Triticum aestivum L.) germplasm using microsatellite markers. Theoretical and Applied Genetics, 105: 699-107. 
10. Jobson, J.D. 1992. Applied multivariate data analysis, Volum П, Categorical and multivariate methods. New York, Springer-Verlag.

11. Kearsey, M.J. and H.S. Pooni. 1996. The genetical analysis of quantitative traits. Chapman and Hall, London.

12. Kimber G. and M. Feldman. 1987. Wild Wheat: An Introduction. Special Report, College of Agriculture, University of Missouri-Columbia.

13. Maleki, M., M.R. Naghavi, H. Alizadeh, P. Potki, M. Kazemi, S.M. Pirseyedi and F. Tabatabaei. 2006. Study of genetic variation in wild diploid wheat (Triticum boeoticum) from Iran using AFLP markers. Iranian Journal of Biotechnology, 4: 269-274 (In Persian).

14. Maric, S., M. Bede, J. Martincic and V. Guberac-Sjemenarstvo. 1998. Variability of some winter wheat traits from breeding process. Siemenarstvo, 15: 421-433.

15. Marić, S., S. Bolarić, J. Martinčić, I. Pejić and V. Kozumplik. 2004. Genetic diversity of hexaploid wheat cultivars estimated by RAPD markers, morphological traits and coefficients of parentage. Plant Breeding, 123: 366-369.

16. Mohammadi, S.A. and B.M. Prasanna. 2003. Analysis of genetic diversity in crop plants-Salient statistical tools and considerations. Crop Science, 43: 1235-1248 (In Persian).

17. Moradi Sarab Shelly, A. 2011. Genetic diversity of wild wheat species using morphological traits. Sciences Journal of plant Production, 34: 364-254 (In Persian).

18. Naghavi, M.R. and R. Amirian. 2005. Morphological Characterization of Accessions of Aegilops tauschii. International Journal Agriculture and Biology, 7:392-394 (In Persian).

19. Naghdipoor, A., M. Khodarahmi, A. Porshahbazi and M. Esmaeilzadeh. 2011. Factor analysis for grain yield and other traits in durum wheat. Journal of Agronomy and Plant Breeding, 7: 84-96 (In Persian).

20. Qualset, C.O. and P.L. Spagnoletti Zeuli. 1987. Geographical diversity for quantitative spike characters in a world collection of durum wheat. Crop Science, 41: 217-235.

21. Romesburg, H.C. 1990. Cluster analysis for researchers. Krieger Publishing, Malabar, Florida.

22. Sears, E.R. 1954. The Aneuploids of Common Wheat. (Anonymous).

23. Shafaoddin, S. and B. Yazdi-Samadi. 1994. Genetic and geographical in Indigenous wheat collection of central Iran. Iranian Journal of Agricultural Science, 25: 61-77 (In Persian).

24. SPSS-Inc. 2013. IBM SPSS statistics 22 core system user's guide. USA: SPSS Inc., an IBM Company Headquarters.

25. Stepien, L., V. Mohler, J. Bocianowski and G. Koczyk. 2007. Assessing genetic diversity of Polish wheat (Triticum aestivum) varieties using microsatellite markers. Genetic Resources and Crop Evolution, 54: 1499-1506.

26. Taleei, A. and B. Behram-Nejad. 2003. A Study of relationship between yield and its components in landrace populations of wheat from western parts of Iran using multivariate analysis. Iranian Journal of Agricultural Science, 34: 949-959 (In Persian).

27. Van Slageren, M.W. 1994. Wild Wheats: A Monograph of Aegilops L. and Amblyopyrum (Jaub. and Spach) Eig (Poaceae). Netherlands Wageningen Agricultural University Papers .

28. Welling, M. 2005. Fisher linear discriminant analysis. University of Toronto Publications, Canada.

29. Zaharieva, M., A. Dimov, P. Stankova, J. David and P. Monneveux. 2003. Morphological diversity and potential intrest for wheat improvement of three Aegilops L. species from Bulgaria. Genetic Resources and Crop Evolution, 50: 507-517.

30. Zergani, M., G.A. Ranibar and S. Ebrahimnezhad. 2015. Molecular assessment of genetic diversity among bread wheat (Triticum aestivum L.) doubled haploid lines using SSR markers. Journal of crop breeding, 7: 88-95 (In Persian).

31. Zohary, D. and M. Hopf. 2001. Domestication of Plants in the Old World. 3rd Edition, Oxford University Press, Oxford. 


\title{
Grouping of Different Populations of Wild Wheat (Triticum Boeoticum) by Multivariate Analysis
}

\author{
Masoud Hamedi ${ }^{1}$, Mahmood Maleki ${ }^{2}$, Mahdi Rahimi ${ }^{3}$, Amin Baghizadeh ${ }^{4}$ and \\ Najmeh Sadat Alavi \\ 1- M.Sc. Student, of Plant Breeding, Faculty of Sciences and Modern Technologies, Graduate University of Advanced \\ Technology, Kerman, Iran \\ 3 and 4- Assistant Professor and Associate Professor, of Plant Breeding, Department of \\ Biotechnology, Institute of Science and High Technology and Environmental Science, Graduate University of \\ Advanced Technology, Kerman, Iran \\ 2- Assistant Professor of Plant Breeding, Department of Biotechnology, Institute of Science and High Technology and \\ Environmental Science, Graduate University of Advanced Technology, Kerman, Iran \\ (Corresponding author: maleki.li@gmail.com) \\ Received: October 27, 2005 \\ Accepted: April 9, 2016
}

\begin{abstract}
27 different populations of Triticum boeoticum were gathered from west and North West of Iran for their grouping using morphological traits. All populations were assessed in farm based on completely random design with three replications in 1393. The measured traits include stem length with spike, spike length with and without awn, awn length, flag leaf length, the woolly leaves, peduncle length, spikelet length, number of grains per spikelet and 100-grain weight. The result showed that the most diversity were observed in hairy leaf and flag leaf length traits according to the variation coefficient in studied traits. All populations are located in three groups based on cluster analysis. Obtained grouping was not agreed with geographical grouping and showed more diversity into populations gathered from the same province. Based on factor analysis, three factors were recognized that explain 76.65 percent of total variation. The first factor allocation of 30.09 percent of the total variation is mainly explained by the traits of hairy leaf, spike length with awn, awn length, grain number per spikelet and was named as a spike morphology factor. The second factor is explained 23.12 percent of the total variation and named length factor and third factor allocation 23.12 percent of the total variation and name were grain filling factor. Biplot figure based on two first factor, all populations were placed on three groups. The obtained results from factor analysis were conformed the result of cluster analysis and confirmed them. The result indicated that when two first factors legitimized high percent of variation, can be used for grouping.
\end{abstract}

Keywords: Cluster analysis, Factor analysis, Wild wheat 